\title{
Tendencias de segregación residencial socioeconómica: el caso de Córdoba (Argentina) en el período 2001-2008
}

Cecilia Marengo. Universidad Nacional de Córdoba, Córdoba, Argentina. Ana L. Elorza. Universidad Nacional de Córdoba, Córdoba, Argentina.

RESUMEN | Los estudios sobre segregación han tomado relevancia en las últimas décadas como consecuencia de los cambios derivados del modelo de acumulación capitalista y sus efectos en las ciudades. El objetivo del artículo es identificar en el municipio de Córdoba (segunda ciudad de Argentina en tamaño) el grado de mixtura socioeconómica que presentan las diferentes áreas urbanas y analizar su evolución en el período 2001-2008, caracterizado por la recuperación económica y la mejora en los indicadores sociales. Se busca aportar un diagnóstico de situación respecto de la segregación residencial socioeconómica en la ciudad, que pueda ser de utilidad para orientar el desarrollo de políticas urbanas y de vivienda al momento de evaluar las posibles localizaciones, así como la escala de los programas habitacionales, factores importantes cuando se trata de acciones tendientes a disminuir las desigualdades sociales y posibilitar la integración de los habitantes en la ciudad.

PALABRAS CLAVE | segregación, pobreza, localización.

ABSTRACT | Segregation studies have become relevant in recent decades as a result of the changes derived from the capitalist accumulation model and its effects on cities. The aim of this paper is to identify in Córdoba municipality (Argentina's second largest city) the degree of socioeconomic mix present in different urban areas and analyze its evolution in the 2001-2008 period, which coincides with economic recovery and improvement in social indicators. It seeks to provide a situation analysis regarding socioeconomic residential segregation in the city -which can be useful to guide the development of urban and housing policies when evaluating possible locations-, and the scale of programs. Both are important factors when it comes to actions aimed to reduce social inequalities and enable the integration of people in the city.

KEY WORDS | segregation, poverty, location.

Recibido el 2 de marzo de 2012, aprobado el 20 de febrero de 2013

E-Mail: Cecilia Marengo, mcmarengo@gmail.com | Ana Elorza, analauraelorza@hotmail.com 


\section{Introducción}

El tema de la segregación residencial ha sido abordado con distintas perspectivas teóricas, iniciándose -entre otros- con los aportes de Robert Park en el Departamento de Sociología de la Universidad de Chicago durante las primeras décadas del siglo pasado. En los años noventa, a partir de la constatación de los impactos del modelo de acumulación capitalista en las estructuras urbanas de las ciudades, se desarrolló un nuevo interés en torno a este fenómeno. Distintos investigadores (Harvey, 2000; Soja, 2000; Davis, 2006; De Mattos, 2010, Borja, 2007, entre otros) destacan entre los nuevos rasgos de las metrópolis, la metropolización extendida, la fragmentación espacial, el aumento de las desigualdades sociales y la profundización de la segregación residencial.

Si bien las ciudades latinoamericanas se caracterizaron desde su conformación por ser segregadas (Ziccardi, 2001; Kaztman, 2001; Duhau, 2003) -es decir, los diferentes grupos sociales siempre se han aglutinado en áreas urbanas (barrios) que se diferenciaban entre sí por su particular nivel socioeconómico, separándose especialmente los sectores de altos ingresos del resto de la población-, en los últimos años este fenómeno toma importancia, debido a que el patrón de la segregación residencial está cambiando. Según Sabatini, Cáceres y Cerda (2001), estas transformaciones se refieren a la escala geográfica y el aumento de la malignidad, esto es, la profundización de las consecuencias negativas en la calidad de vida material y simbólica de la población pobre segregada ${ }^{1}$. Aunque no existe una abultada evidencia empírica que verifique esta hipótesis (Rodríguez \& Arriagada, 2004), se presentan avances en estudios de caso que aportan a ratificar esta tendencia (Kaztman, 2001; Sabatini 2004; Sabatini \& Cáceres, 2005; Smolka, 2003; Queiroz Ribeiro, 2005).

En relación con el primer cambio, los autores plantean que la escala geográfica de la segregación muestra tendencias contradictorias en las ciudades de América Latina; es decir, en un sentido parece disminuir y en otro, aumentar. En ambos casos influyen factores ligados al mercado de suelo. La primera tendencia, positiva, que Sabatini y Cáceres (2005) señalan para el caso de Santiago de Chile, se refiere a la relativa dispersión de los conjuntos residenciales cerrados, los espacios comerciales y los centros de oficinas "modernos" fuera de las áreas tradicionales de concentración de los grupos de altos ingresos. Esta localización es posible gracias a la liberalización de los mercados de suelo, la afluencia y concentración del capital inmobiliario y el mejoramiento de la infraestructura urbana, en especial la infraestructura vial. Es decir, se están acortando las distancias físicas entre los grupos sociales y su localización urbana, aunque esto no signifique una mayor integración entre los mismos o una disminución de las desigualdades sociales. La segunda tendencia, en este caso negativa, corresponde a la intensificación de la segregación residencial socioeconómica que estaría adquiriendo una escala regional, ya que las familias de bajos ingresos que acceden a la vivienda deben hacerlo en localizaciones alejadas de los límites urbanos, en terrenos sin infraestructura ni equipamiento, y en muchos casos son impulsadas fuera de las ciudades centrales hacia localidades del área metropolitana que ofrecen mayores posibilidades de acceso al suelo (de bajo costo) y menores exigencias en términos de requerimientos de urbanización. La mayor escala agudiza los efectos de aislamiento físico, laboral y social de los habitantes y contribuye a la segregación subjetiva, es decir, ayuda a que aumente la sensación de “estar de más” (Sabatini \& Cáceres, 2005). 
En Argentina, desde la década de los noventa se estudia el impacto que sobre la configuración urbana tienen los barrios cerrados de sectores sociales de altos ingresos (denominados "countries"), la fragmentación espacial-urbana y el aumento de la segregación residencial socioeconómica en la ciudad de Buenos Aires (Prevot Shapira, 2002). En los últimos años, ese interés se ha ampliado hacia la medición de dicho fenómeno y su manifestación en distintas ciudades del interior del país. El informe del Programa de Naciones Unidas para el Desarrollo (PNUD, 2009) referido a la segregación residencial en Argentina, analiza sus manifestaciones durante el periodo intercensal 1991-2001 en cuatro áreas urbanas -Ciudad de Buenos Aires, Conurbano bonaerense, Rosario, Gran Córdoba y Mendoza- y demuestra una tendencia al agrupamiento territorial de sectores sociales con bajo nivel de estudio y condiciones laborales precarias.

Por otra parte, Groisman (2011) hace un análisis del comportamiento de la segregación residencial socioeconómica en el país durante los años 2002 a 2007, donde sostiene que a pesar de las mejoras de los indicadores macroeconómicos ${ }^{2}$, el patrón de segregación residencial no se ha modificado de manera sustantiva. Para el autor, ello se debería a que la demanda laboral fue más intensa para los sectores con mayor nivel educativo y que el aumento de las remuneraciones en los empleos de baja calificación no condujo a un sustantivo aumento de la equidad. Concluye, de esta manera, que el crecimiento económico es insuficiente para corregir las desigualdades asociadas a la segregación residencial.

Estudios relativos a la ciudad de Córdoba a escala intraurbana (Marengo, 2004, 2008) demuestran que en esta ciudad existen zonas segregadas por el nivel socioeconómico de la población. En el período intercensal 1991-2001, el fenómeno de la segregación residencial se profundizó en áreas periféricas, aumentando la distancia geográfica entre sectores poblacionales de alto ingreso localizados en el sector noroeste de la ciudad y la población de bajo ingreso localizada en el sector sureste de la misma. En esta última localización se registró la mayor cantidad de viviendas sociales subsidiadas para sectores pobres (implementadas a partir de programas de vivienda pública, como son los programas de erradicación de villas de emergencia). A lo largo de este proceso, donde se observa que se intensifican los niveles de segregación residencial en el área administrativa correspondiente al municipio de Córdoba ${ }^{3}$, han tenido un importante rol las acciones estatales en materia habitacional (tanto municipales como provinciales). La manera de afrontar la problemática de la informalidad urbana y el tipo de programas habitacionales desarrollados, han contribuido a consolidar áreas monofuncionales en la periferia urbana, sin mixtura social ni físico-espacial.

2 De acuerdo con la Comisión Económica para América Latina y el Caribe (CEPAL), la tasa media anual de desempleo descendió de 19,2 en 2002 a 8,5 en 2007 en Argentina. Véase su Anuario Estadístico de América Latina y el Caribe 2010 (www.cepal.org).

3 La ciudad de Córdoba, cabecera del Departamento Capital de la provincia homónima, tiene un ejido urbano de 572 kilómetros cuadrados. Según datos de la Dirección de Urbanismo de la Municipalidad de Córdoba, al año 2010 la superficie urbanizada bruta ascendía a 23.972 hectáreas y el área urbanizable (tierra nueva por urbanizar), a 2.419 hectáreas. 
Continuando con el estudio de la dinámica de este fenómeno en el municipio de Córdoba, en el presente trabajo analizamos su comportamiento durante los años 2001 a 2008, midiendo el nivel de homogeneidad/heterogeneidad en la localización de los grupos sociales en la ciudad. La investigación busca responder el siguiente interrogante: ¿qué dinámica ha tenido el fenómeno de la segregación residencial socioeconómica en la ciudad de Córdoba en el periodo 2001-2008? Este análisis tiene como objetivo aportar un diagnóstico de situación respecto de la segregación residencial socioeconómica en la ciudad, el cual puede ser una herramienta para orientar el desarrollo de políticas urbanas de intervención en el territorio. Específicamente, puede ser útil al momento de evaluar las posibles localizaciones de planes de vivienda, la escala de los programas y la composición social de la población demandante a la que van dirigidos los programas, factores importantes cuando se trata de acciones tendientes a disminuir las desigualdades sociales y posibilitar una mayor integración de los habitantes en la estructura urbana.

La primera sección del artículo aborda el concepto de segregación residencial socioeconómica desde una perspectiva teórica latinoamericana. En segundo lugar, se presenta la situación socioeconómica de la región, y se contextualiza a Argentina y a la ciudad de Córdoba, que observa una notable mejoría en los indicadores sociales relativos a las condiciones de vida de la población. En tercer lugar, se describe la estrategia metodológica empleada para la medición de la segregación residencial socioeconómica en el análisis empírico. Posteriormente se presenta el caso estudiado -la situación de la segregación residencial socioeconómica en el área administrativa del municipio de Córdoba durante el periodo 2001-2008-, con un análisis de la varianza y la desviación estándar, según las variables de segmentación socioeconómica seleccionadas (Nivel de estudios del jefe de hogar y población con Necesidades básicas insatisfechas, NBI). Por último, se aportan conclusiones preliminares sobre la dinámica de este fenómeno en la ciudad.

\section{Aproximación conceptual para el abordaje de la segregación residencial}

En la bibliografía especializada en cuestiones urbanas, en muchas oportunidades se hace referencia de manera indistinta a procesos de división social del espacio, y a segregación social y residencial. Duhau (2003), por su parte, establece una diferenciación entre división social del espacio y segregación residencial. Entiende por la primera, las diferencias existentes en la localización intraurbana o intrametropolitana de diferentes grupos, estratos o clases sociales, relacionadas fundamentalmente con el mercado inmobiliario (costo del suelo y la vivienda), que no son el producto de la exclusión forzada o explícitamente buscada en relación con otros grupos sociales determinados. El segundo caso, la segregación residencial, comprende la segregación urbana y social como un proceso de división social del espacio promovida mediante medidas coercitivas, ya sea a través de políticas o prácticas de exclusión (guetos en Estados Unidos, el apartheid en Sudáfrica, etcétera).

Más allá de parecernos válida esta diferenciación con fines analíticos, en este trabajo consideramos la segregación residencial socioeconómica como un fenómeno que consiste en una relación espacial, que atańe a la proximidad territorial o 
separación entre personas pertenecientes a un mismo grupo socioeconómico (Sabatini, 2004, p. 279), situación que puede estar relacionada con el mercado de suelo y vivienda o con otras prácticas, coercitivas o no.

La segregación residencial presenta tres dimensiones: i) el grado de concentración espacial de los grupos sociales (en términos de localización urbana); ii) la homogeneidad existente en las diferentes áreas de la ciudad en términos de su composición social; y iii) la percepción que las personas tienen de la segregación, como fenómeno espacial. Sabatini (2004) explica que las dos primeras dimensiones hacen referencia al aspecto objetivo de la segregación, aunque cada una capta una arista distinta del fenómeno, mientras que la tercera dimensión se relaciona con aspectos subjetivos. Esta última es importante, dado que se relaciona con la identidad y con el prestigio asignados a barrios o zonas en la ciudad, y contribuye a la construcción de estigmas territoriales con efectos en términos de las posibilidades de integración social de los residentes en esas áreas.

Vignoli (2001) refiere que en las dos primeras dimensiones hay una cierta superposición, ya que la localización de un grupo determinado en una cierta área implica la configuración de un territorio homogéneo; sin embargo, la distinción entre estas dos dimensiones - concentración espacial y homogeneidad social de la población residente- tiene sentido. La primera dimensión tiene lugar cuando algún grupo social registra un sesgo residencial global, es decir, todos (o la gran mayoría) de sus miembros se localizan en una zona determinada del territorio urbano, sin importar si en dicha zona hay otros grupos sociales. Esta segregación por localización de grupo opera cuando, en una situación en la que hay varios grupos sociales, uno (o más) no está disperso en el territorio, sino que se encuentra concentrado en una zona específica. En cambio, la segunda dimensión, que puede denominarse por exclusión, atañe a la ausencia de mezcla o integración de grupos sociales en espacios comunes. En esta dimensión de la segregación, un grupo social no se mezcla con el resto aunque esté diseminado en varias localizaciones de la ciudad. Así se originan zonas homogéneas en un contexto heterogéneo, lo que probablemente dificultará la interacción con otros grupos sociales.

Distintos estudios (por ejemplo, Kaztman, 2001; Sabatini, 2004; Sabatini \& Cáceres, 2005; Smolka, 2003; Queiroz Ribeiro, 2005), analizan diferentes factores que intervienen en la producción del fenómeno señalado, entre los cuales se destacan la flexibilización laboral, el acceso al mercado de suelo urbano, las nuevas pautas residenciales de los grupos de altos ingresos, los estigmas hacia los sectores pobres y los efectos del "aislamiento de los pobres". Además, los trabajos mencionados dan cuenta de que la segregación residencial socioeconómica afecta a los grupos de bajos ingresos e incide en las capacidades y oportunidades que estos tienen para el acceso a una calidad de vida urbana digna. De allí la importancia de identificar los efectos de la segregación residencial en la estructura urbana, los problemas asociados a la localización de las áreas segregadas y las posibles políticas de atención a dichas problemáticas, que contribuyan a romper el círculo de reproducción de la pobreza. 


\section{El contexto socioeconómico: los indicadores sociales en la región, en la última década. El caso de la ciudad de Córdoba, Argentina ${ }^{4}$}

Durante la última década, los países de América Latina han presentado importantes avances en el crecimiento de su economía y en la evolución del empleo. A pesar de que la distribución del ingreso en la región es reconocida por estar entre las más desiguales del mundo, característica que se ha mantenido constante a lo largo de los últimos cuarenta años, en la mayoría de los países ha surgido en los últimos años una incipiente tendencia hacia una menor concentración de la riqueza.

Según CEPAL (2010), entre 2002 y 2006-20085, la brecha entre quintiles extremos de la distribución se redujo en 14 países sobre un total de 18, y el índice de Gini bajó por lo menos en un 5\% en 11 países. Este proceso de disminución de la concentración de la riqueza es acompañado por lo general con la reducción de los niveles de pobreza e indigencia. En comparación con el año 2002, ambos indicadores han mostrado una reducción importante, de 10,9\% el primero y $6,1 \%$ el segundo.

En Argentina, como corolario del régimen de convertibilidad guiado por las premisas neoliberales, estalló en el ańo 2001 la mayor crisis económica y social de la historia del país. La política monetaria de devaluación produjo una situación de default, acompańado por una caída del PBI de 16\%, la subida de la tasa de desocupación al 21\% y la disminución del salario en un 24\% de las remuneraciones reales (CEPAL, 2002), lo que repercutió en la agudización de las situaciones de pobreza e indigencia. Datos de la Encuesta Permanente de $\operatorname{Hogares}^{6}$ (EPH) del Instituto Nacional de Estadísticas y Censos (INDEC) demuestran que, en mayo de 2002, la proporción de personas en situación de indigencia en el país era de 24,8\% y la pobreza alcanzaba al $53 \%$ de la población.

A partir de las presidencias de Néstor Kirchner (2003) y de Cristina Fernández (2007) comenzó un proceso de transformación del modelo de desarrollo. En esos años, la política macroeconómica estuvo orientada a promover políticas de valor de cambio competitivo, lo cual significó la recuperación de la industria y del mercado interno y la expansión del modelo extractivo-exportador. Según un informe del Centro de Investigación y Formación de la República Argentina ${ }^{7}$ (CIFRA, 2011), la nueva orientación macroeconómica permitió un crecimiento de la economía argentina superior al registrado en las principales economías latinoamericanas (Brasil y Chile), pasando la tasa de crecimiento anual de 2,7\% del PBI en el periodo 1991-2001, al 7,4\% en el período 2002-2010, alza que fue acompañada por la recuperación del empleo y la disminución de la tasa de desocupación al 7,3\%. También las tasas de pobreza e indigencia presentaron una evolución favorable, disminuyendo la población en situación de pobreza a $17,8 \%$ y de indigencia, a $5,1 \%$.

Los datos de este apartado fueron presentados en el xxvir Congreso Internacional de la Asociación Latinoamericana de Sociología, 6 al 11 de septiembre de 2011, Universidad Federal de Pernambuco (ufpe), Recife. Grupo de Trabajo No 2, "Ciudades latinoamericanas en el nuevo milenio".

5 Según cada país, los datos disponibles refieren a encuestas más recientes. Solo República Dominicana y Guatemala tuvieron un deterioro distributivo en el periodo (CEPAL, 2010).

6 Datos referidos a conglomerados urbanos.

7 Este centro forma parte de la Central de Trabajadores de Argentina (CTA). 
La reactivación económica y las políticas implementadas han tenido como resultado una mejor distribución de los ingresos, tendiente a disminuir la concentración de la riqueza. Según cepal, el coeficiente de Gini que en el año 2002 era de 0,58, disminuyó a 0,51 en el año 2006.

Estos cambios provienen de distintas interacciones entre el crecimiento del ingreso medio de las personas (efecto crecimiento) y los cambios en la forma en que se distribuye ese ingreso (efecto distribución). Cabe destacar que en el grupo de 18 países de la región, Argentina se encuentra, en materia distributiva, por debajo de Uruguay, Perú, El Salvador, Ecuador y Costa Rica (CEPAL, 2010).

La ciudad de Córdoba (capital de la provincia de Córdoba) es reconocida como la segunda ciudad con mayor cantidad de habitantes del país después de la Ciudad Autónoma de Buenos Aires y registra, según el Censo Nacional de 2010, 1.315.423 personas. La dinámica económica y las condiciones de vida de la población en la ciudad, en el periodo estudiado, han acompañado las tendencias nacionales. El panorama crítico del 2001 (Cuadro 1) fue mejorando, registrándose una disminución de la población en situación de pobreza, indigencia y desempleo.

Cuadro 1 | Gran Córdoba. Población en situación de pobreza, indigencia y desempleo (2001-2008) (porcentajes)

\begin{tabular}{|l|c|c|}
\hline \multicolumn{1}{|c|}{ DATOS GRAN CóRDOBA } & AÑO 200I (\%) & AÑo 2008 (\%) \\
\hline Hogares en condiciones pobreza & 24,70 & 14,80 \\
\hline Hogares en condiciones indigencia & 7 & 2,60 \\
\hline Tasa de desempleo & 15,90 & 7,60 \\
\hline
\end{tabular}

FUENTE ELABORACIÓN PROPIA CON DATOS DE INDEC, EPH PARA EL AGLOMERADO CÓRDOBA

En relación con el nivel de concentración de la riqueza, el coeficiente de Gini para la ciudad de Córdoba tiene un valor inferior al nacional $(0,431)$, lo que indica menores desigualdades sociales en cuanto a percepción de ingresos, comparativamente con los valores nacionales (Red Ciudadana Nuestra Córdoba, 2011, p. 66).

En síntesis, en el período analizado todos los indicadores demuestran una importante recuperación de la economía y mejores condiciones de vida para la población. No obstante, las dificultades de acceso al suelo urbano y la vivienda por parte de los grupos sociales de menores ingresos continúan siendo significativas. Un relevamiento realizado por la ONG "Un techo para mi país" (2011) indica que, si bien durante este período no ha habido un número significativo de nuevos asentamientos informales, se registra en el $62 \%$ de los ya existentes un fuerte crecimiento del número de familias en situación de pobreza (período 2001-2011). La modalidad de acceso al suelo en las villas de emergencia y los asentamientos es a través de prácticas desarrolladas en el marco del mercado inmobiliario informal ${ }^{8}$. Este crecimiento continuo no solo se vincula con los altos costos de vida (alquileres, comestibles, transportes) y el valor de vivienda a quien los ocupaba previamente; otra estrategia consiste en que una persona (o grupo de personas), que residen o no en el asentamiento, toman terrenos y, guiados por la lógica de la ganancia, venden las tierras en su propio beneficio. 
los inmuebles y/o terrenos, que se tornan inasequibles para parte de la población, sino también con el proceso de inmigración de población de países cercanos, como Paraguay, Bolivia y Perú. Según el Censo provincial (2008), en la ciudad residen 48.357 inmigrantes; por lo general, estas personas arriban a Córdoba por razones laborales y conforman nuevos asentamientos informales, con la perspectiva de alcanzar su regularización en el largo plazo, como es el caso de los asentamientos de Ampliación Pueyrredón, Renault, Ampliación Nuestro Hogar, entre otros. La persistencia de los asentamientos y su crecimiento continuo dan cuenta del carácter estructural de este fenómeno aun en contextos económicos favorables.

\section{Medición de la segregación residencial socioeconómica: aspectos metodológicos}

Cabe destacar que comprender la segregación residencial socioeconómica y enfocar su análisis implica, desde luego, aplicar una metodología específica para su medición. Uno de los índices más utilizados en los estudios referidos a esta temática es el índice de disimilaridad. El mismo permite calcular la proporción de residentes de un determinado grupo social que tendría que mudar su lugar de residencia para que su presencia relativa en cada subárea considerada en la escala de análisis sea uniforme, es decir, se observen valores similares de mixtura social. Sin embargo, varios autores destacan ciertas limitaciones de este índice para medir la segregación residencial socioeconómica y seńalan que no captura la esencia espacial del fenómeno, al reducir las relaciones a promedios y/o porcentajes; al no captar la segregación al interior de las áreas de medición (por ejemplo, áreas censales), tampoco lo hace a escala mayor (lo que se conoce como el problema del "tablero de ajedrez"). Se señala, además, que el valor del índice cambia en relación con la modificación del área analizada: mientras más pequeña sea esta, el valor del índice aumenta ("problema de la grilla”); funciona con grupos sociales discretos; representa una pérdida de información cuando se quiere estudiar la segregación socioeconómica (no racial o étnica); y por último, este índice entrega un solo valor resumen para toda la ciudad (Vignoli, 2001; Sabatini, 2004; Rodríguez \& Arriagada, 2004; etcétera) ${ }^{10}$.

Para el estudio que planteamos respecto de la segregación residencial socioeconómica en el área administrativa del municipio de Córdoba durante el periodo 2001-2008, nos interesa identificar el nivel de homogeneidad/heterogeneidad que presenta la localización de los diferentes grupos sociales en el espacio. El análisis de

Se caracterizan por su localización periférica y dispersa en la estructura urbana y demandan políticas de regularización para poder acceder a la infraestructura y servicios básicos. Como ejemplo del crecimiento significativo de estos asentamientos se menciona el caso del denominado Nuestro Hogar, donde en 1997 se registraban 100 familias, que para 2001 se habían incrementado a 500 familias y en 2010 alcanzaban a 1.600 familias.

10 Si bien existen antecedentes de trabajos de investigación basados en el cálculo del Índice de Disimilitud (Díaz \& Caro, 2002, citado por Tecco \& Valdez, 2007, que dan cuenta de que para el año 2001 la ciudad de Córdoba era segregada), debido a las críticas mencionadas sobre los resultados de este tipo de índice, consideramos necesario avanzar en otro tipo de análisis estadístico que considere la complejidad del fenómeno. 
esta dimensión a escala de fracciones censales ${ }^{11}$ nos permite detectar directamente las áreas urbanas que presentan alta homogeneidad respecto de los grupos que las habitan. Para realizar esta medición recurrimos al análisis estadístico de la varianza y la desviación estándar, calculando la media de las variables seleccionadas (en este caso, Nivel de estudio del jefe de hogar y población con Necesidades básicas insatisfechas, NBI) e indicadores de dispersión (varianza y desviación estándar) que sugieren el grado de homogeneidad/heterogeneidad (Rodríguez \& Arriagada, 2004, p. 10).

Debido a que los Censos Nacionales de población y vivienda en Argentina no incorporan indicadores relativos de segregación, en especial el ingreso de los hogares, para su medición definimos como variables de segmentación socioeconómica los años de estudio de jefe de hogar y las necesidades básicas insatisfechas ${ }^{12}$ (NBI por persona), las cuales nos permiten determinar la pertenencia social de la población a diferentes grupos. Esta definición se basa en el supuesto de que existe una correlación entre la educación del jefe de hogar y la probabilidad de obtener mayores ingresos familiares; $y$, por lo tanto, un mejor acceso a satisfactores de las necesidades básicas. El análisis se realiza con datos secundarios: el Censo de Población Nacional del año 2001 realizado por el Instituto Nacional de Estadísticas y Censos (INDEC) y el Censo de Población Provincial de 2008 efectuado por la Dirección de Estadística y Censo de la provincia de Córdoba.

El procedimiento de medición de la segregación desarrollado en la investigación se divide en dos instancias: primero, se ha calculado la media de años de estudios de los jefes de hogar para toda la ciudad y el valor de desviación de cada unidad geográfica (fracciones censales) respecto del promedio de la ciudad para los dos cortes temporales (2001 y 2008). Con esos resultados, se realiza una clasificación en cuatro intervalos, según la intensidad de la desviación estándar de la variable analizada por fracción. Es decir, se considera, en un primer intervalo, aquellos valores de desviación estándar comprendidos entre el 1\% y el 10\% (por encima y por debajo de la media), lo cual seńala heterogeneidad en la distribución y, consecuentemente, un bajo valor de segregación. Un segundo intervalo, con valores comprendidos entre el $11 \%$ y el $20 \%$ (por encima y por debajo de la media ${ }^{13}$ ), los cuales se consideran valores moderados de segregación. El tercer intervalo incluye valores comprendidos entre el $21 \%$ y el $30 \%$ (por encima y por debajo de la media), que se consideran valores altos de segregación. Finalmente, el cuarto intervalo abarca valores que superan el 30\% (por encima y por debajo de la media), que se consi-

11 El Departamento Capital, que coincide con el área administrativa del municipio de Córdoba, se divide en 84 fracciones censales; estas, a su vez, en 1.377 radios censales; y estos, en segmentos, que son las unidades de análisis asignadas a cada censista.

12 Se consideran personas con NBI aquellas que presentan al menos uno de estos indicadores de privación: pertenecientes a hogares con más de tres personas por cuarto; habitantes de una vivienda de tipo inconveniente; residentes en viviendas sin ningún tipo de retrete; personas que tuvieran algún niño en edad escolar (6 a 12 años) que no asistiera a la escuela; y hogares con cuatro o más personas por miembro ocupado y, además, cuyo jefe no haya completado tercer grado de escolaridad primaria. Véase Observatorio Urbano de la Ciudad (s/f), Barrios bajo la lupa. Cap. 2: "Aspecto social", p. 3. En http://bit.ly/1c4IX6o

13 Los valores por encima de la media se asocian con sectores sociales de ingresos medio altos/alto, y los valores por debajo de la media con sectores sociales de ingresos medio bajos/muy bajos. 
deran de muy alta segregación. Estos intervalos han sido localizados en un plano de la ciudad (Figuras 1 y 2), a los efectos de identificar los sectores urbanos en situación de mayor homogeneidad/heterogeneidad territorial, considerando esta variable de segmentación socioeconómica.

En segunda instancia, realizamos el cómputo para la medición según la variable NBI. Se ha calculado la media de población con NBI para la ciudad y el valor de la varianza de cada fracción, identificando la incidencia del valor de cada fracción en el total de población con NBI de la ciudad (Cuadro 2). Una vez obtenidos estos datos, seleccionamos las fracciones donde la cantidad de población con NBI supera a la media de la ciudad; y a los fines analíticos, clasificamos los valores en cuatro intervalos según la intensidad de la varianza obtenida por fracción. Los mismos se identificaron como: nivel bajo de segregación (con variación del 1\% al 10\%), nivel moderado de segregación (con variación entre 11\% y 20\%), nivel alto de segregación (con variación entre $21 \%$ y $30 \%$ ), y nivel muy alto de segregación (con variación de más del 30\%). Estos resultados también han sido espacializados en el plano de la ciudad (Figuras 3 y 4) y superpuestos con los resultados del análisis previo obtenido al analizar la variable Nivel de estudio del jefe del hogar. Esta lectura comparada ha permitido identificar las fracciones censales (áreas) ${ }^{14}$ de la ciudad donde se evidencia una mayor homogeneidad en términos de población en situación de pobreza y con bajo nivel de estudio.

\section{Evolución de la segregación residencial en Córdoba en el período 2001-2008}

El análisis de la segregación residencial en el municipio de Córdoba es una herramienta importante al momento de planificar la localización de intervenciones habitacionales implementadas mediante políticas públicas. Consideramos que el conocimiento sobre el grado de mixtura socioeconómica presente en las diferentes áreas de la ciudad (y su evolución) puede ser de utilidad al momento de evaluar las posibles localizaciones de planes de vivienda, específicamente en el caso de aquellos conjuntos destinados a población vulnerable (bajos ingresos), dado que la localización (o relocalización) en un determinado lugar será un factor importante, en cuanto condiciona las posibilidades de acceso a las oportunidades laborales, educativas y servicios urbanos. En buena medida determinará los niveles de integración física y social de los habitantes en su nueva localización.

Por otra parte, la concentración de sectores de alto ingreso en una determinada localización urbana está contribuyendo a promover las ventajas comparativas de esas áreas, en términos de desarrollo económico, vitalidad urbana, oportunidades para la localización comercial y de servicios, valorización del suelo, entre otros aspectos. Como contrapartida, existen en la ciudad áreas deprimidas, tugurizadas, con bajos niveles de equipamientos y servicios, que no logran capturar inversiones o retener

14 Si bien el estudio se realiza a escala de fracciones censales, que es la unidad de medición censal, es de mencionar que las mismas no coinciden necesariamente con la delimitación de los barrios que integran la ciudad. Es decir, desde el punto de vista de la intervención urbanística, sería necesario, en futuros estudios, ajustar estos resultados del análisis a la delimitación físico-espacial considerada como unidad de intervención en la planificación urbana. 
condiciones de la mixtura social de los habitantes y requieren de intervenciones en materia de políticas públicas para revertir esta condición.

Para nuestro análisis, tuvimos en cuenta la hipótesis de Jaramillo (1999), según la cual las formas particulares de segregación en las ciudades latinoamericanas deben ser explicadas mediante el análisis del mercado de suelo urbano y de las posibilidades que los diferentes grupos sociales tienen para elegir una determinada ubicación. Sobre esa base, hemos podido identificar cómo evolucionaron los indicadores de segregación residencial socioeconómica en la ciudad de Córdoba entre los periodos 1991 y 2001 (en el contexto de la orientación neoliberal de la década de los noventa en Argentina) y 2001 y 2008, que se caracteriza por la recuperación económica.

Estudios realizados sobre segregación residencial en la ciudad de Córdoba en el período 1991-2001 (Marengo, 2008) evidencian que hacia el final del mismo se intensifican los niveles de segregación residencial. Acompañando el proceso de polarización de la estructura social, numerosas áreas urbanas (consideradas a escala de fracciones censales) pierden heterogeneidad socioeconómica y la mixtura social que tenían a comienzos de la década de los noventa. En el período analizado, la varianza (medida por el nivel de educación del jefe del hogar ${ }^{15}$ ), incrementa su valor de 0,035 a 0,053, ampliándose la brecha entre las áreas habitadas por sectores de altos ingresos y aquellas en que residen familias de bajos ingresos. Los valores de desviación se incrementan de 0,44 a 0,49 hacia las fracciones censales donde se localizan los sectores de mayores ingresos, y de 0,21 a 0,26 en las fracciones censales donde se localizan los sectores de menores ingresos. Estos valores señalan una mayor homogeneidad social en el espacio, en términos de Vignoli (2001), que podemos referir como intensificación de la segregación por localización de grupo.

La intensificación de la segregación a escala de la ciudad se relaciona con el patrón de crecimiento urbano que se observa en este período, caracterizado por la extensión del tejido residencial y la incorporación de nuevas áreas con una composición social homogénea en la periferia. En el período analizado aumenta la distancia geográfica entre la localización de la población de altos ingresos (hacia el eje norte de expansión urbana) y la población de bajos ingresos (en el sector sur-sureste de la ciudad).

La forma de crecimiento que prevalece en este período (extensión dispersa) reproduce condiciones de alta inequidad espacial. Las zonas intermedias de la ciudad que cuentan con provisión de infraestructura y servicios urbanos y mayor heterogeneidad social pierden población, mientras que en los bordes urbanos se incrementa la cantidad de población residente. La relación entre expansión urbana y segregación residencial pone en evidencia un aumento de la distancia espacial y social de los diferentes grupos de población y la consolidación de áreas socialmente homogéneas en los bordes de la ciudad.

Hacia 2008, el análisis de la segregación residencial evidencia que la misma disminuye. Acompañando el crecimiento económico y la mejora en la distribución de ingresos, antes señalada, aumentan los sectores de la ciudad que presentan una mayor heterogeneidad en cuanto a la composición de los grupos sociales

15 La media de años de estudio de jefes de hogar registra un incremento progresivo en los diferentes cortes intercensales; para el Censo 1991, era de 8,89 años; se incrementó a 9,64 en el Censo 2001 y a 11,7 ańos de estudio en el Censo 2008. 
residentes. La varianza (medida por el nivel de educación del jefe del hogar) disminuye de 0,053 (en 2001) a 0,039 (en 2008). La brecha entre las áreas que habitan sectores de alto y bajo ingreso se acorta, lo cual señala una desaceleración del proceso de segregación de población, con homogeneidad social en el espacio. Los valores de desviación disminuyen de 0,49 a 0,45 en las fracciones censales donde se localizan preferentemente los sectores de mayores ingresos, y de 0,26 (en 2001) a 0,19 (en 2008) en las fracciones censales donde se localizan en su mayoría los sectores de menores ingresos. La espacialización de estos valores se representa en las Figuras 1 y 2.

FIGURA 1 | Segregación residencial socioeconómica en la ciudad de Córdoba, 2001

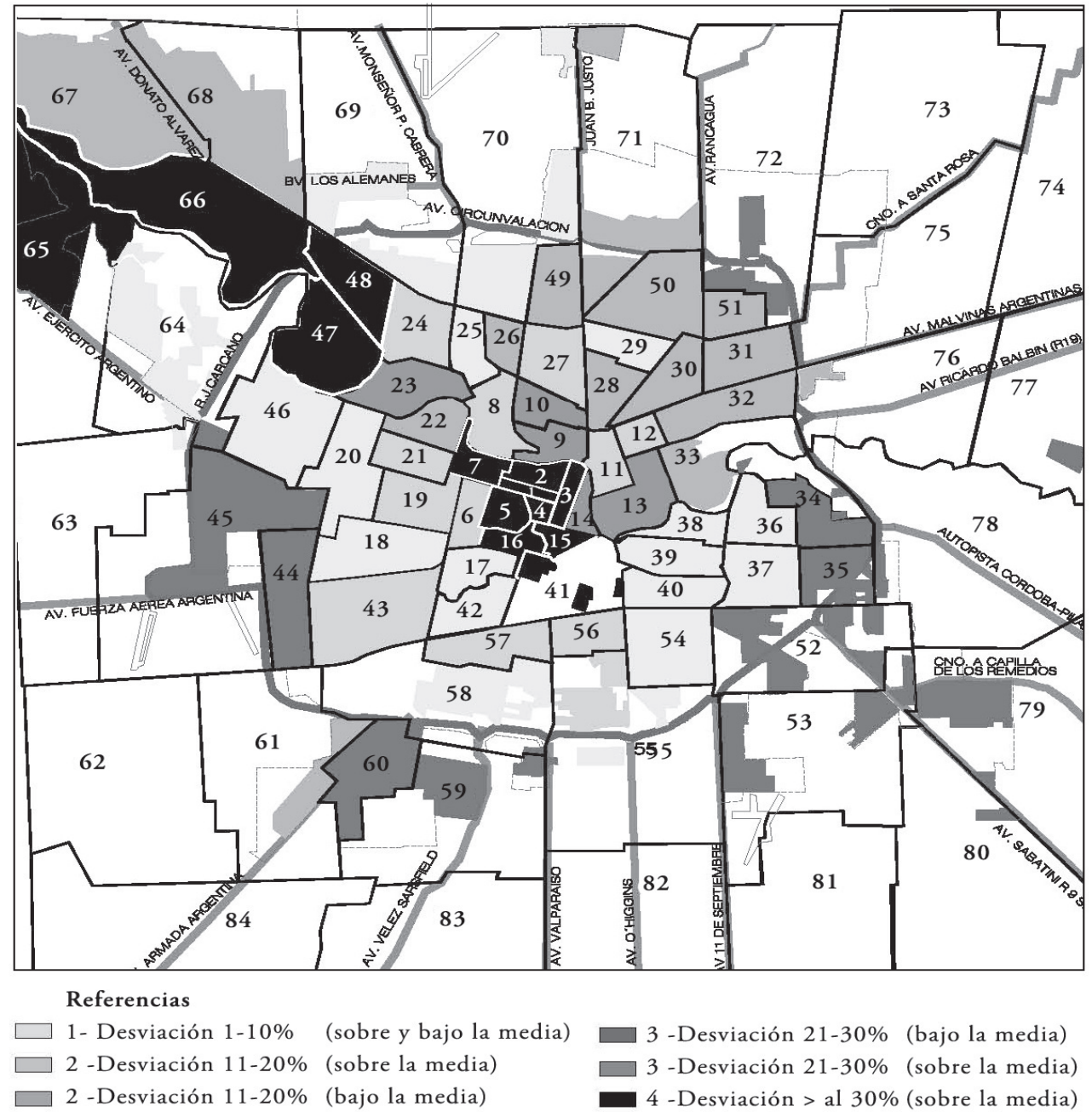

FUENTE ELABORACIÓN PROPIA, A PARTIR DE CENSO 200 I 
FIGURA 2 Segregación residencial socioeconómica en la ciudad de Córdoba, 2008

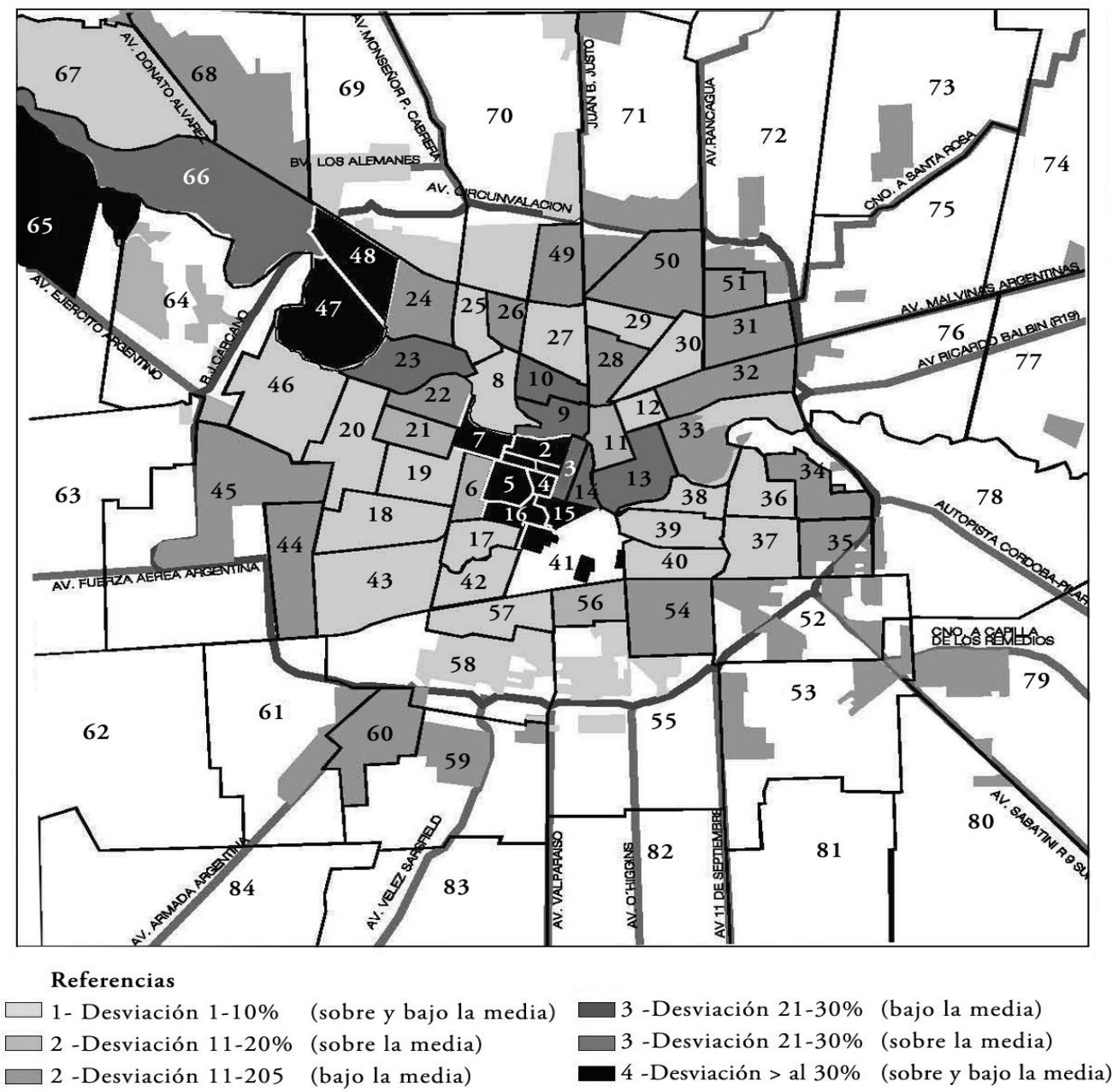

FUENTE ELABORACIÓN PROPIA, A PARTIR DE CENSO 2008

Análisis de la segregación residencial considerando como indicador las NBI El estudio de la variación de la variable NBI es importante para describir la distribución que presentan los habitantes con pobreza estructural. Si bien -como se analizó en el apartado anterior- la pobreza ha disminuido, y en el período 2001-2008 la población de la ciudad de Córdoba con NBI se redujo de 155.319 habitantes a 128.966, persisten áreas que se caracterizan por ser fuertemente segregadas. A los efectos de identificar las áreas donde hay una localización de población en situación de pobreza estructural en el municipio de Córdoba y analizar su evolución, se examina la segregación residencial desde la perspectiva de este indicador.

El análisis de la pobreza urbana mediante la medición de necesidades básicas insatisfechas muestra que, a escala de ciudad, la varianza disminuye de 25,77 en 2001 a 20,99 en 2008. Esta disminución alcanza a numerosas fracciones censales en las que se registra la depreciación de las varianzas entre las subunidades territoriales que las integran. Sin embargo, y a pesar de la disminución en valores absolutos de 
la pobreza estructural en la ciudad de Córdoba, si consideramos las áreas específicas (a escala de fracciones censales) donde habita la población con NBI, observamos que la misma presenta una baja reducción: de $33,33 \%$ en 2001 a 32,14\% en 2008; es decir, la población con NBI continúa localizándose en áreas acotadas de la ciudad.

A los efectos de facilitar la lectura de los valores para este indicador, los agrupamos en cuatro intervalos de análisis (Cuadro 2):

a. Nivel bajo de segregación (que incluye los valores de variación entre 1\% y 10\%). En el periodo analizado se registra un incremento en la cantidad de fracciones con bajo nivel de segregación. Los valores generales para este intervalo han disminuido de $46,15 \%$ a $40,74 \%$. La dinámica de este patrón de segregación ha sido variada; encontramos fracciones (por ejemplo las números 32 y 33) que en 2001 presentaban valores de varianzas de 13,15 y 19,38 respectivamente, y que en 2008 han decrecido a 8,39 y 4,28, lo que se interpreta como una menor localización de población con NBI en dichas áreas.

b. Nivel moderado de segregación (que incluye los valores de variación entre $11 \%$ y 20\%). En este intervalo, comparativamente con 2001, se ha incrementado la cantidad de fracciones que lo integran, y los valores se modifican de 10,71\% a $14,81 \%$. En este caso se presentan situaciones opuestas. Por un lado, fracciones que en 2001 presentaban niveles moderados de segregación y que en 2008 han aumentado la población en situación de pobreza estructural (como en el caso de la fracción 49, donde aumenta la población con NBI de 2.615 a 2.860 habitantes); y por el otro, fracciones donde hay una disminución en el valor de la varianza, es decir, presentan una menor homogeneidad social, como es el caso de las fracciones $32,33^{16}$ y 53 .

c. Nivel alto de segregación (con valores de varianza entre $21 \%$ y $30 \%$ ). Este grupo se ha mantenido cuantitativamente constante, registrándose en ambos períodos solo una fracción con valores comprendidos en este intervalo. En 2001, la fracción 46, con un valor de 28,37; y en 2008, la fracción 60, que tiene un valor de 29,33.

d. Nivel muy alto de segregación (con valores de varianza de más del 30\%). También este patrón se mantiene con moderados cambios. Puede afirmarse que, en términos generales, disminuye la cantidad de fracciones con altos valores de población con NBI, observándose valores de variación de 42,86\% en 2001 a 40,74\% en 2008. Sin embargo, es llamativo el crecimiento de población con NBI en la fracción 72, habiendo aumentado su varianza de 10,25 a 37,87 (lo que representa un aumento de 3.111 a 3.745 habitantes).

16 Estas fracciones, por la dinámica de su varianza respecto del NBI, han cambiado de categoría. En 2001 (Figura 3) se incluían en el intervalo "nivel moderado de segregación”, y en 2008 (Figura 4) se encuentran en el intervalo "nivel bajo de segregación". 


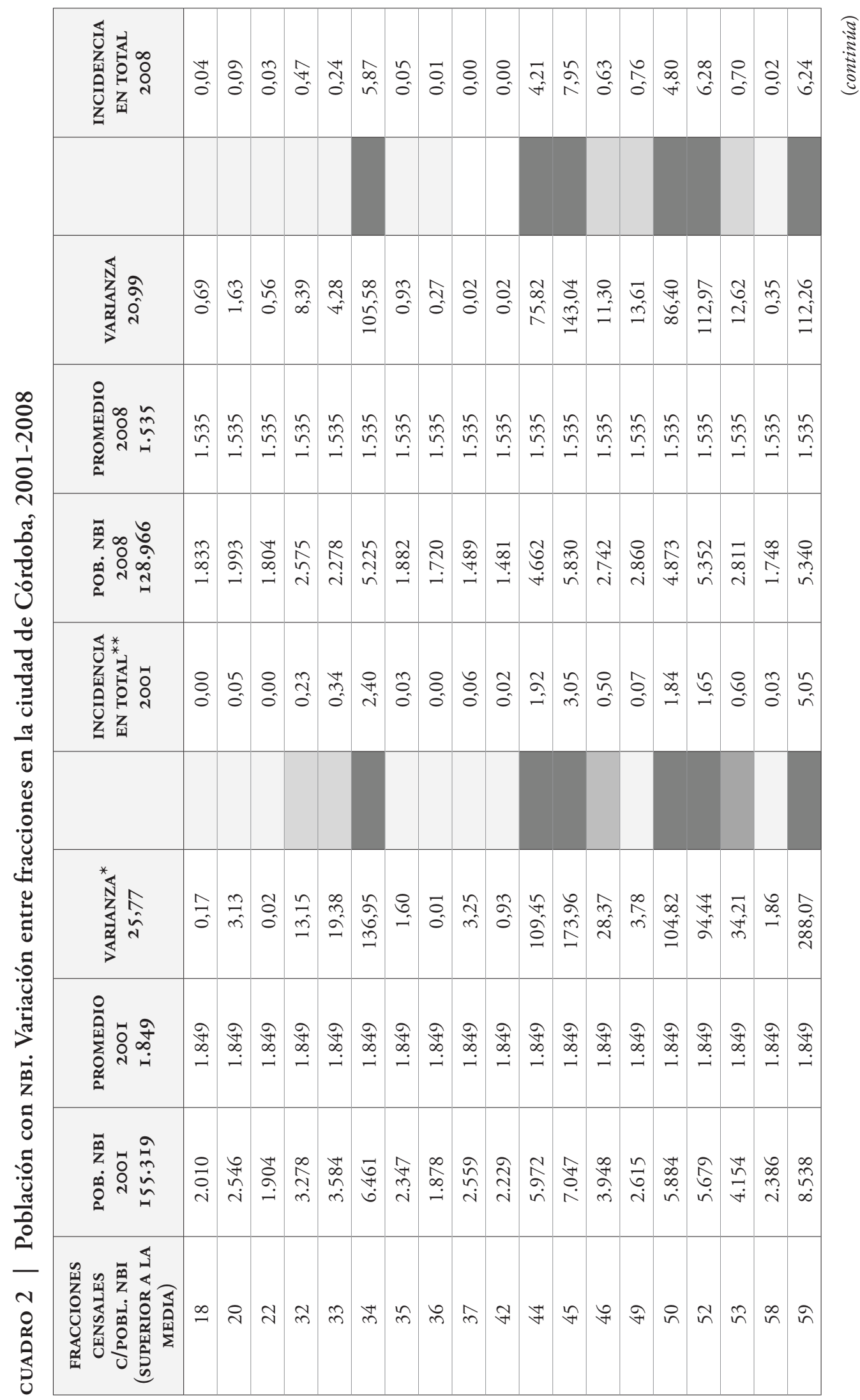




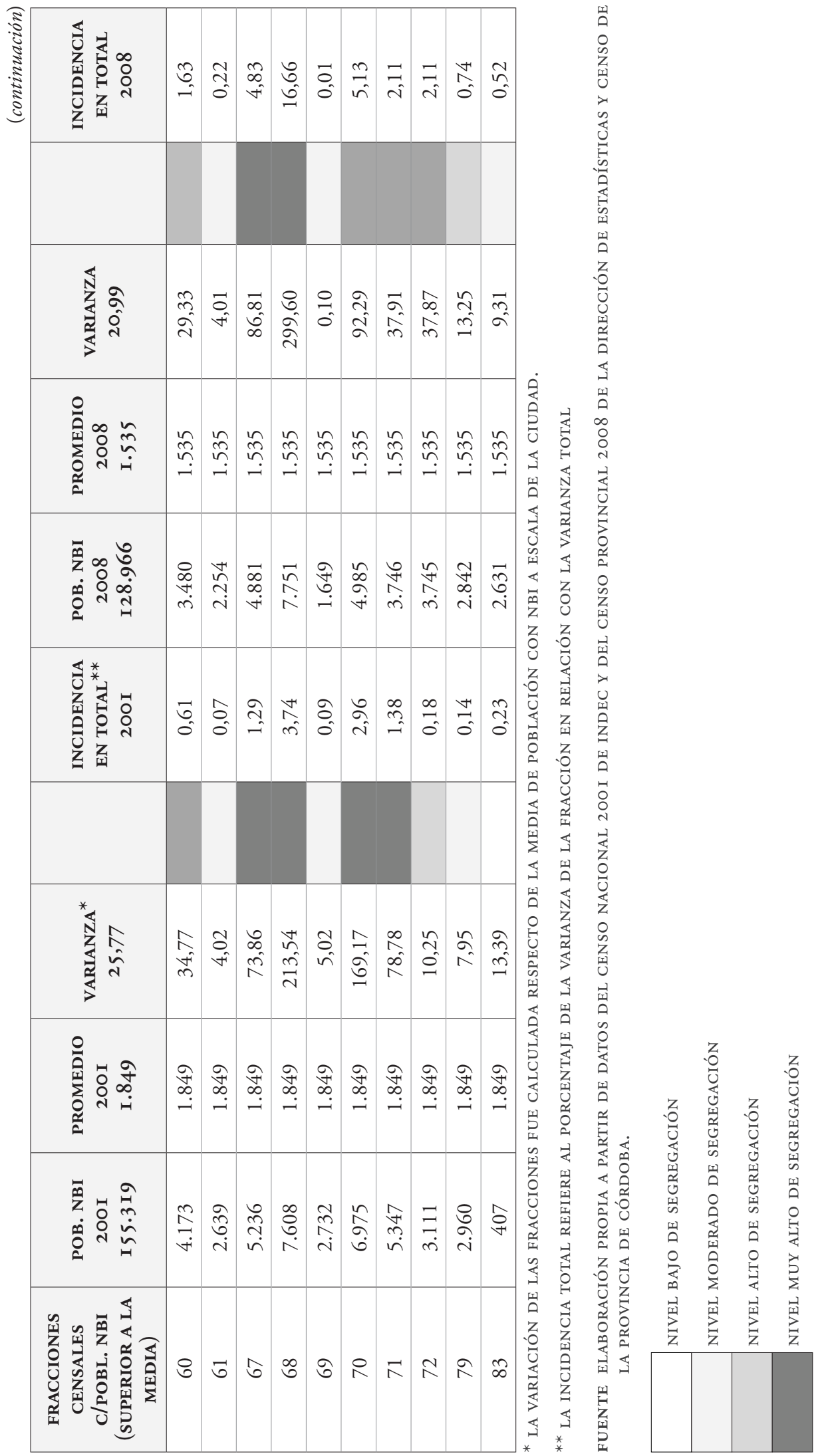


Como puede observarse en un análisis comparado (2001-2008), la distribución de la población en situación de pobreza estructural (señalada por círculos, rombos, etcétera, en las Figuras 3 y 4) mantiene una tendencia de localización en determinadas áreas periféricas de la ciudad, coincidentes con los intervalos de desviación que presentan niveles bajos y medios de segregación (comprendidos entre el 11\% y $20 \%$, y el $21 \%$ y $30 \%$ bajo la media, que corresponden a los sectores de bajos ingresos). A pesar de la disminución de la población con NBI respecto de los valores de 2001, puede afirmarse que la localización urbana de este grupo y su distribución en el espacio no ha variado de manera significativa. Existen áreas en la ciudad que se caracterizan por registrar valores que se mantienen relativamente constantes en términos de localización por grupos en situación de pobreza estructural, intensificándose, en consecuencia, los niveles de segregación residencial en un contexto económico favorable.

Se observa una desigual distribución de la pobreza estructural en la ciudad de Córdoba en áreas localizadas en los bordes urbanos, históricamente segregadas. Mientras que, en 2001, el 62,42\% de la población con NBI vivía en la periferia de la ciudad, en el 2008 este porcentaje había aumentado a 65,43\% (aun cuando se registra una disminución de habitantes con NBI, de 96.964 a 84.324). Es decir, en términos generales hubo una mejora en los condiciones de vida, pero en el caso particular de la población en condiciones de pobreza estructural, las desigualdades persistían en la última fecha medida. Es de mencionar que en algunas fracciones censales se registran valores muy altos de localización de población con NBI, comparativamente con lo que sería un valor de distribución media para la ciudad. (Por ejemplo, las fracciones números 34, 45, 52, 59 y 68 registran 5.225, 5.830, 5.352, 5.340 y 7.751 habitantes con NBI, respectivamente, cuando el valor de distribución media es de 1.535 habitantes.)

Esta localización en determinados puntos del espacio urbano periférico refuerza, en el período analizado (2001-2008), la presencia de población pobre en áreas de la ciudad históricamente pobres; y señala, por otra parte, la imposibilidad de acceso para estos habitantes a localizaciones más consolidadas en la estructura urbana. Este proceso de segregación residencial socioeconómica es producto de la lógica del mercado de suelo urbano, que no solo afecta las posibilidades de acceso a la vivienda por parte de los habitantes pobres, las cuales quedan reducidas sea a la instalación en áreas donde los valores de la tierra son bajos y la infraestructura y servicios públicos son deficitarios, sea a las tomas de tierras. Además, dicha lógica plantea interrogantes sobre las acciones que en materia habitacional desarrolla el Estado para estos grupos poblacionales. En el caso del municipio de Córdoba, al no existir una política de suelo que pueda atenuar los efectos de un mercado especulativo, la localización de conjuntos habitacionales para población vulnerable desarrollados por el Estado a través de políticas públicas queda sujeta a las mismas condicionantes de acceso al suelo que cualquier iniciativa privada. La alta especulación sobre el suelo urbano y la inexistencia de reservas de tierra para la construcción de conjuntos de vivienda social, obliga a localizar los planes en áreas donde el suelo es barato (anexando parcelas de suelo rural que se urbanizan y se incorporan como suelo urbanizado). Cuando a esta condición se suma la falta de mixtura social de los destinatarios de las operatorias 
habitacionales, al estar orientadas las acciones de vivienda a una demanda social homogénea (situación que ha prevalecido en la implementación de políticas habitacionales focalizadas), se reduce la posibilidad de mixtura y de heterogeneidad social en las nuevas áreas residenciales y se contribuye a consolidar sectores urbanos fuertemente segregados ${ }^{17}$. De esta forma, la política social de vivienda pública intensifica los procesos de segregación, con la localización de población vulnerable en áreas donde persisten condiciones de pobreza (círculo vicioso), condicionando las posibilidades de una efectiva integración de los habitantes a la vida urbana en contextos que debiera promover la heterogeneidad social (círculo virtuoso).

FIGURA 3 | Segregación residencial socioeconómica en la ciudad de Córdoba según NBI, 2001

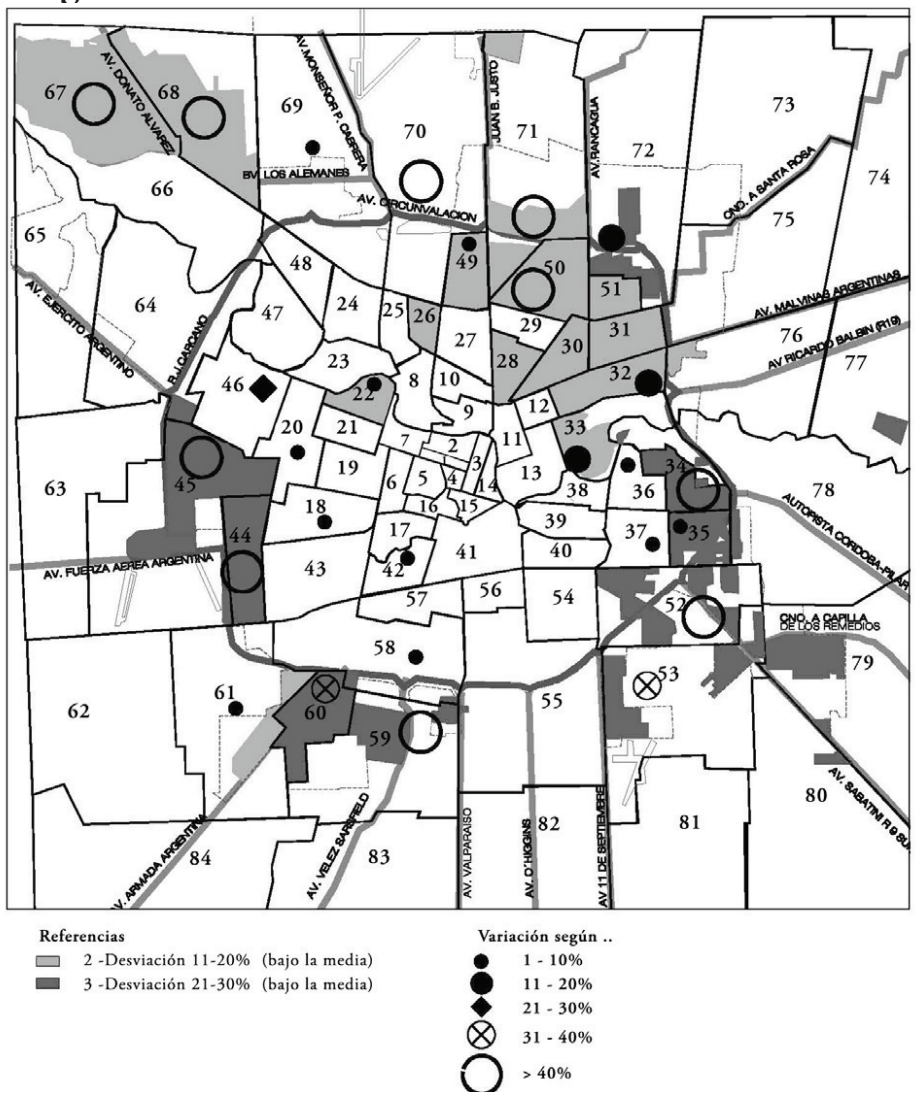

FUENTE ELABORACIÓN PROPIA, A PARTIR DE CENSO 200 I

17 En la ciudad de Córdoba, desde el año 2003 hasta 2010, se ha desarrollado un programa de relocalización de asentamientos informales a nuevos barrios, en el marco del cual se han trasladado aproximadamente 70 asentamientos a 41 nuevos barrios, de los cuales 11 son denominados como "barrios-ciudad", diferenciados por su escala, que supera las 500 viviendas. La localización de los conjuntos habitacionales configura una nueva distribución de la población pobre en la ciudad: ha sido trasladada desde el anillo central e intermedio a los anillos periféricos; por lo general se la localiza en áreas de extensión urbana, la mayor parte de las veces incorporando suelos que estaban afectados al uso rural (Marengo \& Elorza, 2011). Resultados de diversas investigaciones estarían demostrando que esta política ha contribuido a la ruptura de redes laborales y de ayuda mutua de las familias relocalizadas (Montoya Flores, 2006) y a la reproducción de pobreza en estos nuevos barrios (Elorza, 2009). 
FIgURA 4 Segregación residencial socioeconómica en la ciudad de Córdoba según NBI, 2008

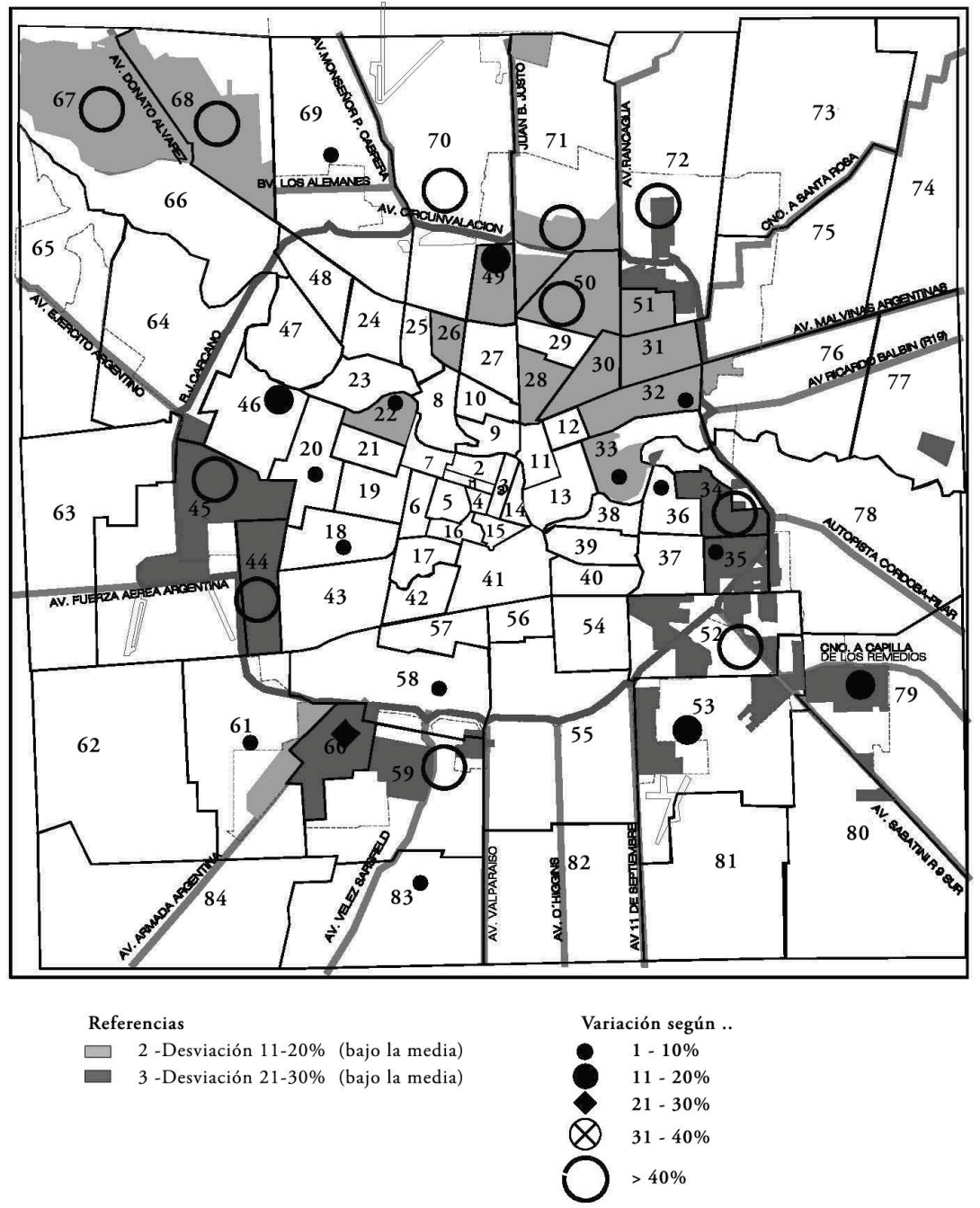

FUENTE ELABORACIÓN PROPIA, A PARTIR DE CENSO 2008

\section{Conclusiones}

El caso de Córdoba, Argentina, evidencia que la escala geográfica de la segregación muestra tendencias contradictorias, como ya lo mencionaban los estudios de Sabatini, Cáceres y Cerdá (2001) para el caso de Santiago de Chile. Es decir, cuando la escala geográfica considerada es toda la ciudad, los resultados indican que en el período analizado tiene lugar una desaceleración de la segregación residencial, observándose un acortamiento en la distancia social de los diferentes grupos poblacionales y una mayor heterogeneidad en la composición de la población, en la mayoría de los sectores analizados. La comparación del análisis de la segregación en la ciudad de Córdoba en el período 2001-2008 evidencia que esta se atenúa, modificando la tendencia a la intensificación que se había registrado en el período 1991-2001. En términos físico-espaciales, se observa que las áreas con desviación 
(bajo la media) agrupada en el intervalo 11\%-20\% disminuyen significativamente, y en amplios sectores de la ciudad los valores de desviación están comprendidos entre el $1 \%$ y el $10 \%$, es decir, en el intervalo que indica baja segregación.

Los resultados obtenidos, a escala de la ciudad, indican una estrecha vinculación entre las condiciones de desigualdad de la población y la segregación residencial socioeconómica, en tanto localización de los distintos grupos en el territorio. En el ańo 2001, los indicadores sociales de las condiciones de vida de la población daban cuenta de altos porcentajes de pobreza y dificultades para la continuidad en el sistema educativo, acompañados con una profundización de la segregación residencial socioeconómica. A la inversa, para 2008, cuando estos indicadores reflejan una importante recuperación y avances en las condiciones de vida y en el nivel de estudios de la población, también la medición de la segregación residencial socioeconómica da cuenta de una disminución en ella.

Sin embargo, cuando se considera el análisis desde la perspectiva del indicador Necesidades básicas insatisfechas a nivel de fracciones censales, se observa que dichas carencias se intensifican en determinadas áreas localizadas en la periferia urbana, donde se registran históricamente altos valores de segregación residencial. Es decir, si centramos el análisis en los grupos más vulnerables, observamos una tendencia al agrupamiento en determinados sectores de la periferia urbana, intensificándose de esta manera las condiciones de homogeneidad social en el espacio. Es de observar que en algunas de estas áreas los valores de localización de población en situación de pobreza se incrementan, aun cuando la tendencia que se observa en este período es a la disminución de población con NBI, dadas las mejoras en términos de crecimiento económico en el país y la orientación de las políticas sociales. Se han intensificado las condiciones de localización de población pobre en áreas históricamente pobres, lo que implica que de no revertirse esta tendencia, la evolución de las mismas puede volverse crítica en el futuro.

Este trabajo representa un primer paso en el complejo estudio del fenómeno de la segregación residencial socioeconómica en la ciudad de Córdoba. Según dijimos, aporta a la realización de un diagnóstico respecto de la división social del territorio y puede contribuir a la planificación de acciones en el espacio urbano. En especial, puede aplicarse en la orientación y el diseño de nuevos programas y formas de intervención que tiendan a disminuir las desigualdades sociales y espaciales, por ejemplo promoviendo una mayor mixtura social en las nuevas áreas residenciales, desarrollando acciones de política urbana que revitalicen áreas que cuentan con una capacidad instalada y pueden albergar mayor población. Apunta también a la necesidad de estudiar posibles programas de densificación habitacional con mixtura social, que pudieran contrarrestar el crecimiento disperso en extensiones periféricas socialmente homogéneas. 


\section{Referencias bibliográficas}

Aliaga-Linares, L. \& Alvarez-Rivadulla, M. (2010). Segregación residencial en Bogotá a través del tiempo y diferentes escalas. Documento de Trabajo de Lincoln Institute of Land Policy. Recuperado de http://bit.ly/19h8zP7

Arriagada, C. \& Rodríguez Vignoli, J. (2003). Segregación residencial en áreas metropolitanas de América Latina: magnitud, caracteristicas, evolución e implicaciones de política. Serie Población y Desarrollo, 47. Santiago, Chile: Cepal / Centro Latinoamericano y Caribeño de Demografía (Celade)-División de Población Proyecto Regional de Población celade / Fondo de Población de las Naciones Unidas (UNFPA). Recuperado de http://bit.ly/1hqTcTR

Borja, J. (2007). Revolución y contrarrevolución en la ciudad global: las expectativas frustradas por la globalización de nuestras ciudades. EURE, 33(100), 35-50. http://dx.doi. org/10.4067/S0250-71612007000300003

Comisión Económica para América Latina y el Caribe (CEPAL), Naciones Unidas. (2010). Panorama social de América Latina. Documento INFORMATIVO. Recuperado de http://www.eclac.org/publicaciones/xml/9/41799/PSE-panoramasocial2010.pdf

Centro de Investigación y Formación de la República Argentina (CIFrA). (2011). El nuevo patrón de crecimiento. Argentina 2002-2010. Informe de Coyuntura, 7 (mayo 2011). Buenos Aires, Argentina. Recuperado de http://es.scribd.com/doc/56731427/CIFrAInforme-de-Coyuntura-07-Mayo-2011

Davis, M. (2006). Planeta de Ciudades Miseria. (J. M. Amoroto Salido, Trad.). Madrid: Foca - Akal.

De Mattos C. (2010). Una nueva geografía latinoamericana en el tránsito de la planificación a la gobernanza, del desarrollo al crecimiento. EURE, 36(108), 167-179. http://dx.doi. org/10.4067/S0250-71612010000200010

Elorza A. L. (2009). Estrategias de reproducción social de familias relocalizadas, entre la adaptación y el cambio. Conciencia Social, 9(12), 53- 59.

Duhau, E. (2003). División social del espacio metropolitano y movilidad residencial. Papeles de Población, 9(36), 161- 211. Recuperado de http://www.redalyc.org/articulo. oa?id=11203608

Groisman, F. (2011). Segregación residencial socioeconómica en Argentina durante la recuperación económica (2002-2007). Documentos de Trabajo 3, Universidad de Alcalá. Instituto de Estudios Latinoamericanos (IELAT). Recuperado de www.red-redial.net/revista-doc umentos, de,trabajo,ielat.218-2011

Harvey D. (2000). Mundos urbanos posibles. En A. M. Ramos (Coord.), Lo urbano en 20 autores contemporáneos (pp. 177-197). Barcelona: Ediciones Universitat Politècnica de Catalunya.

Harvey, D. (2009) El derecho a la ciudad. Programa de Gestión de la Ciudad. Recuperado de http://cafedelasciudades.com.ar/carajillo/1_art5.htm (24/09/2009)

Jaramillo S. (1999). El papel del mercado del suelo en la configuración de algunos rasgos socioespaciales de las ciudades latinoamericanas. Territorios, 2, 107-129 (Universidad del Rosario, Colombia). Recuperado de http://www.redalyc.org/articulo.oa?id=35700206

Kaztman, R. (2001). Seducidos y abandonados: el aislamiento social de los pobres urbanos. Revista de la CEPAL, 75, 171-189. Recuperado de http://www.eclac.cl/publicaciones/ xml/6/19326/Katzman.pdf 
Marengo, C. (2004). Una aproximación a la segregación residencial como punto de partida en la formulación de políticas. Boletín del INVI, 19(50), 165-181 (Universidad de Chile). Recuperado de http://www.revistas.uchile.cl/index.php/InvI/article/viewArticle/8813 Marengo, C. (2008). Urban sprawl and planning: Facing the challenges of growing social inequity. Case study: Cordoba - Argentina. Tesis doctoral, publicada por International Forum on Urbanism (IFoU), The Netherlands.

Marengo C. \& Elorza, A. (2009). Globalización y políticas urbanas. La política habitacional focalizada como estrategia para atenuar condiciones de pobreza urbana: los programas implementados en Córdoba y los desafíos pendientes. Cuaderno Urbano, 8, 7-33. Recuperado de http://dialnet.unirioja.es/servlet/articulo?codigo $=4081566$

Marengo C. \& Elorza A. (2011). Políticas habitacionales y Segregación Residencial en Córdoba (Argentina) en el contexto neoliberal. Presentación en XXVII Congreso Internacional de la Asociación Latinoamericana de Sociología, 6 al 11 de septiembre de 2011, Universidad Federal de Pernambuco (UfPE), Recife. Grupo de Trabajo No 2, "Ciudades latinoamericanas en el nuevo milenio".

Montoya Flórez, O. (2006). Calidad de vida en un barrio de habitantes erradicados en la periferia de Córdoba. Estudio de caso: barrio Ciudad de mis Sueños. Lineamientos para la gestión ambiental. Trabajo de Tesis Maestría en Gestión Ambiental del Desarrollo Urbano, Facultad de Arquitectura, Urbanismo y Diseño, Universidad Nacional de Córdoba.

Prevot Shapira, M. F. (2002). Buenos Aires en los años 90: metropolización y desigualdades. EURE, 28(85), 31-50. http://dx.doi.org/10.4067/S0250-71612002008500003

Programa de Naciones Unidas para el Desarrollo (PNUD). (2009). Segregación residencial en Argentina. Buenos Aires: Autor.

Queiroz Ribeiro, L. C. (2005). Segregación residencial y segmentación social: el "efecto vecindario" en la reproducción de la pobreza en las metrópolis brasileñas. En S. Álvarez Leguizamon (Comp.), Trabajo y producción de la pobreza en Latinoamérica y el Caribe: estructura, discursos y actores. (pp. 137-155). Buenos Aires: Consejo Latinoamericano de Ciencias Sociales (CLACSO).

Red Ciudadana Nuestra Córdoba. (2011). Indicadores ciudadanos Nuestra Córdoba 2011. Córdoba, Argentina. Recuperado de http://www.nuestracordoba.org.ar/documentos/ Indicadores_Nuestra-Cba_2011.pdf

Rodríguez Vignoli, J. (2001). Segregación residencial socioeconómica: ¿qué es?, ¿Cómo se mide?, ¿qué está pasando?, ¿importa? Serie Población y Desarrollo, 16. Santiago, Chile: Comisión Económica para América Latina y el Caribe (CEPAL). Recuperado de http:// www.eclac.cl/publicaciones/xml/8/7888/lcl1576-P.pdf

Sabatini, F. (2003). La segregación social del espacio en las ciudades de América Latina. Banco Interamericano de Desarrollo, Departamento de Desarrollo Sostenible, División de Programas Sociales. Recuperado de http://idbdocs.iadb.org/wsdocs/getdocument. aspx?docnum $=1442235$. También publicado en Serie Azul, 35. Santiago: Documentos del Instituto de Estudios Urbanos y Territoriales, Pontificia Universidad Católica de Chile.

Sabatini, F. (2004). Medición de la segregación residencial: reflexiones metodológicas desde la ciudad latinoamericana. En G. Cáceres \& F. Sabatini (Eds.), Barrios cerrados en Santiago de Chile: entre la exclusión y la integración residencia (pp. 277-307). Santiago, Chile: Lincoln Institute o Land Policy / Universidad Católica de Chile, Instituto de Geografía. 
Sabatini, F. \& Cáceres, G. (2005). ¿Es posible la mezcla social en el espacio?: la inclusión residencial y la nueva conflictividad urbana en Latinoamérica. Documento de Lincoln Institute of Land Policy. Recuperado de www.territorioysuelo.org/documentos

Sabatini, F., Cáceres, G. \& Cerda, J. (2001). Segregación residencial en las principales ciudades chilenas: Tendencias de las tres últimas décadas y posibles cursos de acción. EURE, 27(82), 21-42. http://dx.doi.org/10.4067/S0250-71612001008200002

Smolka, M. (2003). Informalidad, pobreza urbana y precios de tierra. Documento de Lincoln Institute of Land Policy. Recuperado de www.territorioysuelo.org/documentos

Soja, E. (2000). Seis discursos sobre la postmetrópolis. En A. M. Ramos (Coord.), Lo urbano en 20 autores contemporáneos (pp. 91-88). Barcelona: Ediciones Universitat Politècnica de Catalunya.

Tecco, C. \& Valdez, E. (2007). Segregación residencial socioeconómica (sRs) e intervenciones para contrarrestar sus efectos negativos: Reflexiones a partir de un estudio en la ciudad de Córdoba, Argentina. Cuadernos de Geografía, 15, 35-66. Recuperado de http:// www.revistas.unal.edu.co/index.php/rcg/article/viewFile/1286/1831.

Ziccardi, A. (2001). Las ciudades y la cuestión social. En A. Ziccardi (Coord.), Pobreza, desigualdad social y ciudadanía. Los límites de las politicas sociales en América Latina (pp. 85-126). Buenos Aires: Consejo Latinoamericano de Ciencias Sociales (ClACso). Recuperado de http://bibliotecavirtual.clacso.org.ar/clacso/gt/20101029062411/ziccardi.pdf 